\title{
Opinión de los Profesores Españoles del Grado de Ciencias de la Actividad Física o del Deporte sobre la Calidad de las Editoriales Científicas en la Difusión del Conocimiento en las Ciencias del Deporte
}

\author{
Jesús Salado y Enrique Ortega \\ Facultad Ciencias del Deporte, Universidad de Murcia, Campus de Excelencia Mare Nostrum, C/Argentina s/n Santiago \\ de la Ribera-San Javier, Murcia - España. (e-mail: jsaladotarodo@gmail.com; eortega@um.es)
}

Recibido Nov. 27, 2018; Aceptado Ene. 28, 2019; Versión final Mar. 8, 2019, Publicado Ago. 2019

\begin{abstract}
Resumen
El objetivo del presente estudio es conocer la opinión del Personal Docente e Investigador de Ciencias del Deporte (PDI) sobre: a) los criterios que debe tener una editorial para que sea considerada de reconocido prestigio; y b) qué editoriales nacionales e internacionales son las que tienen mayor prestigio. La muestra estuvo compuesta por 142 profesores universitarios españoles que impartían docencia en el grado de Ciencias de la Actividad Física y del Deporte. Los resultados obtenidos en el presente estudio llevan a las siguientes conclusiones: 1) Las editoriales españolas de reconocido prestigio más valoradas por el PDI de Ciencias de la Actividad Física y el Deporte son: Inde, Paidotribo y Wanceulen; 2) Las editoriales internacionales de reconocido prestigio más valoradas por el PDI de Ciencias de la Actividad Física y el Deporte son: Human Kinetics, Elsevier y Taylor and Francis; y 3) Los aspectos más importantes que tiene que tener una editorial para ser considerada de reconocido prestigio son: tener revisores externos, tener comités de lectura especializados y tener una elevada presencia en bases de datos internacionales.
\end{abstract}

\section{Opinion of the Spanish Professor of Sport Science Degree on the Quality of Scientific Editorials in the Transmission of Knowledge in Sports Sciences}

\begin{abstract}
The objective of the present study is to know the opinion of university professors of Sports Sciences (PDI) on: a) the criteria that an editorial must have to be considered as of recognized prestige; and b) what national and international editorials are the most prestigious. The sample was composed of 142 Spanish university professors who teach in the degree of Physical Activity and Sport Sciences. The results obtained in the present study lead to the following conclusions: 1) The Spanish publishers of recognized prestige most valued by the professors of Physical Activity and Sports Sciences are: Inde, Paidotribo and Wanceulen; 2) The prestigious international publishers most valued by the professors of Sports Sciences are: Human Kinetics, Elsevier and Taylor and Francis; and 3) The most important aspects that a publisher must have to be considered as of recognized prestige are: having external reviewers, having specialized reading committees and having a high presence in international databases.
\end{abstract}




\section{INTRODUCCIÓN}

La evaluación del profesor universitario es uno de los aspectos más importantes a tener en cuenta en la evaluación de la calidad de una universidad. Esta evaluación en España la realiza, entre otras agencias, la Agencia Nacional de Evaluación de la Calidad y Acreditación (ANECA) mediante sus programas de evaluación del profesorado para al acceso a las diferentes figuras (PEP, ACADEMIA, CNEA). En el Real Decreto 415/2015, de 29 de mayo (España, 2015), por el que se modifica el Real Decreto 1312/2007, de 5 de octubre, por el que se establece la acreditación nacional para el acceso a los cuerpos docentes universitarios se establecen los siguientes criterios de evaluación: docencia, formación académica, gestión universitaria, transferencia de conocimiento e investigación (RD 415/2015). Con respecto a la evaluación de la docencia se tienen en cuenta aspectos como dedicación docente, calidad de la actividad docente, calidad de la formación docente y otros méritos. Destacar la dificultad de la evaluación del aspecto docente debido a su multidimensionalidad (Codina, 2016).

Otro de los criterios que aparecen en el Real Decreto es la formación académica en la cual se tienen en cuenta aspectos como son: "calidad de la formación predoctoral y doctoral: becas, premios, otros títulos, etc., tesis doctoral: premio extraordinario de doctorado y otros premios, mención de doctorado europeo o internacional, mención de calidad o excelencia del programa de doctorado, calidad de la formación posdoctoral: becas o contratos posdoctorales y otros méritos de formación académica" (p. 50334). El tercer aspecto evaluado es el de la gestión, siendo el menos valorado. Este aspecto gestor es tenido en cuenta también en la mayoría de los países (Buela et al., 2009). Otro aspecto evaluado es la transferencia de conocimiento y experiencia profesional, en el cual se tienen en cuenta los siguientes ítems (RD 415/2015, p. 50334): "Patentes y productos con registro de propiedad intelectual, transferencia de conocimiento al sector productivo, implicación en empresas de base tecnológica, etcétera, calidad y dedicación a actividades profesionales en empresas, instituciones, organismos públicos de investigación u hospitales, distintas a las docentes o investigadoras, contratos de transferencia o prestación de servicios profesionales con empresas, Administraciones públicas y otras instituciones suscritos al amparo del artículo 83 de la Ley Orgánica 6/2001, de Universidades, y otros méritos de transferencia de conocimiento y experiencia profesional".

Por último, en la evaluación de la investigación los aspectos que se valoran son (RD 415/2015, p. 50334): "calidad y difusión de resultados de la actividad investigadora: publicaciones científicas, creaciones artísticas profesionales, congresos, conferencias, seminarios, tesis doctorales dirigidas (si no se alegan como méritos de actividad docente), calidad y número de proyectos competitivos y contratos de investigación con resultados constatables, movilidad: estancias en centros de investigación con resultados constatables y otros méritos investigadores". Esta evaluación del aspecto investigador del profesorado universitario es el apartado más valorado en las distintas acreditaciones existentes. En este sentido, Buela-Casal y Sierra (2007) encuestaron a 1294 profesores universitarios y obtuvieron como resultados que los indicadores con mayor peso eran los referidos a la investigación, en especial los artículos publicados en revistas incluidas en el Journal Citation Report (JCR), incluso en campos de conocimiento como Arte y Humanidades y en Ciencias Sociales y Jurídicas. Por otro lado, Larrán-Jorgeet al. (2013) en las consideraciones finales de su trabajo señalan que la situación de las distintas disciplinas es radicalmente diferente, ya que existen diferencias en cuanto a hábitos de publicación, canales disponibles y oportunidades alternativas. En esta misma línea Giménez-Toledo (2016) en su trabajo indica que los investigadores de Humanidades y Ciencias Sociales hacen llamamientos a considerar que cada disciplina tienen sus propias formas de investigar y sus propias formas de publicar por lo que debería tener sus propias formas de evaluación.

En este sentido y teniendo en cuenta el área de Ciencias Sociales y Jurídicas uno de los medios más utilizado, a la hora de transmitir el conocimiento, son los libros que, a diferencia de las revistas científicas, cuentan con pocos instrumentos e indicadores para evaluar su calidad (Giménez-Toledo, 2016; Giménez-Toledo y Tejada-Artigas, 2015). Entre los indicadores de calidad de los libros más utilizados están SPI (Scholarly Publishers Indicators), Book Citation Index y Publisher Scholar Metrics. Por otro lado, Zuccala, Guns, Cornacchia, y Bod (2015) realizan un ranking de editoriales usando los títulos de libros citados en Scopus (2007-2011) y Metadatos de WorldCat@ (es decir, números OCLC, ISBN, registros de editores y conteos de la biblioteca) como indicadores de calidad. El SPIs es "un sistema de información que aporta indicadores y herramientas relacionados con las editoriales científicas para la investigación en el ámbito de las Humanidades y las Ciencias Sociales. Los indicadores que se incluyen pretenden servir como referencia en los procesos de evaluación y permitir la objetivación de algunos conceptos como el de "prestigio de la editorial" (tomado de http://ilia.cchs.csic.es/SPI/proyecto.html). Estos se agrupan en tres bloques de información e indicadores propios: a) rankings de prestigio de editoriales académicas españolas y extranjeras como resultado de una encuesta masiva a investigadores en 16 áreas de conocimiento; b) información sobre especialización temática de las diferentes editoriales; y c) información sobre los sistemas de evaluación de los originales que las editoriales declaran utilizar (Giménez-Toledo et al., 2015). Con respecto a este indicador y para validarlo Giménez-Toledo y Mañana-Rodríguez (2017) utilizan el método Delphi, llegando a la conclusión que después de dos consultas, la encuesta del SPI y este mismo trabajo, hay consenso y poca polarización de los resultados en las primeras posiciones del ranking. 
El Book Citation Index es la base de datos de libros de Thomson Reuters, basado en datos bibliométricos (Jarvis, 2010). Sobre esta base de datos Torres-Salinas y Delgado-López-Cózar (2013) analizan su cobertura, obteniendo como conclusión que tiene una clara orientación anglosajona con poca cabida para editores de otros países. También realiza una comparación con el SPI, en la cual observan que para España no se adapta a las necesidades de la comunidad científica de Ciencias Sociales. Por otro lado, Publisher Scholar Metrics (Grupo de Evaluación de la Ciencia y la Comunicación Científica (EC3), 2012) "es un índice bibliométrico que pretende medir el impacto de las editoriales de monografías científicas a partir del recuento de citas de los libros publicados por los profesores e investigadores de universidades públicas españolas indizados en Google Scholar hasta 2012 en el ámbito de las Humanidades y Ciencias Sociales. Los indicadores empleados se han basado en los siguientes datos: 1) Número de libros de una editorial que figuran entre los libros altamente citados de la muestra; y 2) Número total de citas de los libros de una editorial que figuran entre los libros altamente citados de la muestra. Los rankings de editoriales del Publisher Scholar Metrics se presentan por disciplinas y áreas de conocimiento a las que están adscritos los profesores universitarios españoles". En esta misma línea, se aprecia como otro indicador utilizado para la evaluación de la ciencia el prestigio de la editorial, si bien no está definido a que se refiere reconocido prestigio. En este sentido en la bibliografía se encuentran algunos trabajos en los cuales se les pregunta a los profesores de distintas materias cuales son los aspectos que ellos creen que debe tener una editorial para considerarla de reconocido prestigio (GiménezToledo y Tejada-Artigas, 2012; Giménez-Toledo y Tejada-Artigas, 2015). En este sentido Giménez-Toledo y Tejada-Artigas (2012) encuestaron a 3045 profesores e investigadores los cuales eligieron características de calidad editorial tener una trayectoria continuada, sistema de evaluación con revisores externos, presencia en bibliotecas especializadas, presencia en bases de datos internacionales de las monografías publicadas por la editorial y editorial con colecciones especializadas presencia en librerías especializadas nacionales y extranjeras.

En España promovido por la Unión de Editoriales Universitarias Españolas (UNE) y avalado por la Agencia Nacional de Evaluación de la Calidad y Acreditación (ANECA) y la Fundación Española para la Ciencia y la Tecnología (FECYT) se ha implantado el sello de calidad en edición académica (sello CEA) que tiene como objetivo (ANECA, 2017) "reconocer las mejores prácticas dentro de la edición universitaria española y convertirse en un signo distintivo que tanto las agencias de evaluación de la actividad investigadora como la comunidad académica e investigadora podrán identificar fácilmente. Pretende igualmente ser una vía para promover y estimular la calidad en la edición académica" (tomado de http://www.selloceaapq.es/), hasta la fecha muy poco o nada valorada por las agencias de evaluación. Los indicadores de calidad usados en esta convocatoria son: identificación de la editorial, disponibilidad electrónica, inclusión de metadatos, identificación del director, comité científico, política editorial, regularidad en la producción, coherencia, instrucciones a los autores, información sobre el proceso de selección de manuscritos, informes de evaluadores, listado de revisores externos, comunicación motivada de la resolución, existencia de reseñas, apertura a autores externos a la institución, atracción de autores extranjeros, presencia en bases de datos.

Con respecto a las Ciencias de la Actividad Física y del Deporte, no hay estudios que determinen qué aspectos deben tener las distintas editoriales para que sean de reconocido prestigio. Además, no existen trabajos específicos que establezcan algún tipo de ranking, sino que generalmente las editoriales de Ciencias del Deporte son incluidas en secciones más genéricas como educación o psicología. En este sentido, y siguiendo trabajos semejantes en otras áreas como el de Giménez-Toledo, Tejada-Artigas, y Mañana-Rodríguez (2012), y con la finalidad de disponer de una elemento más (la propia opinión de los evaluados) que permita una adecuada evaluación, los objetivos que se plantean en este trabajo son: a) Conocer la opinión de los PDI en Ciencias de la Actividad Física y del Deporte sobre los criterios que debe tener una editorial para que sea considerada de reconocido prestigio; b) Realizar un ranking de editoriales nacionales según la opinión de los PDI en Ciencias de la Actividad Física y del Deporte; y c) Realizar un ranking de editoriales internacionales según la opinión de PDI en Ciencias de la Actividad Física y del Deporte.

\section{METODOLOGÍA}

Los participantes fueron 142 profesores universitarios ( $20 \%$ del total) que impartían docencia en el grado de Ciencias de la Actividad Física y del Deporte en el curso 2013-2014 en universidades públicas ( $N=710$ profesores; datos procedentes de la Conferencia Española de Decanos y Directores de Grados de Ciencias de la Actividad Física y el Deporte) de los cuales 75 son funcionarios y 67 son interinos y contratados pertenecientes a 19 Universidades Españolas. La experiencia media en docencia fue de $13.52 \pm 8.12$ años. En España las figuras del profesorado se dividen en dos grandes grupos. En primer lugar, los funcionarios, que son contratados indefinidos por la administración pública, y cuentan con las figuras de profesor titular de escuela, profesor titular de universidad, catedrático de escuela y catedrático de universidad, siendo esta última el máximo rango. Por otro lado, los interinos y contratados, con contratos temporales con la administración pública, y cuentan con las figuras de asociado, ayudantes, ayudante doctor y contratado doctor siendo esta última la de mayor rango de este grupo. 
Con el fin del registrar los datos se usó el Cuestionario sobre "Criterios de Calidad del Personal Docente e Investigador Universitario en Ciencias de la Actividad Física y Deporte (CPDI-CAFD)" (Salado, 2017), instrumento que obtuvo unos valores mínimos de validez de $V$ de Aiken, de 0.81 , y de fiabilidad de 0.93. Del total de ocho bloques que componen el CPDI-CAFD, para el presente estudio se utilizó el apartado de EDITORIALES, en el que se les preguntaba a los PDI que valorasen de 0-5, su opinión sobre 18 ítems en relación a lo que consideran ellos que debe ser una editorial de reconocido prestigio, así como se le solicitaba que indicasen el ítem más importante (la inmensa mayoría de estos 18 ítems, proceden de la traducción y adaptación al ámbito de ciencias del deporte del instrumento diseñado por Giménez-Toledo, Tejada-Artigas, y Mañana-Rodríguez (2012). Finalmente, se les pidió que indicasen las cinco editoriales nacionales e internacionales de mayor prestigio. Para el análisis estadístico de los datos se realizó un análisis descriptivo de la opinión de los PDI calculando la media y desviación típica, así como la frecuencia absoluta y relativa de cada uno de los ítems. Posteriormente, se realizó una comparación de medias mediante la prueba T para muestras independientes y una comparación de proporciones a través de la prueba Chi-cuadrado. En todos los casos se utilizaron un nivel de significación de $p<.05$. Los resultados fueron analizados mediante el paquete estadístico SPSS 21.0.

\section{RESULTADOS}

En la tabla 1 se puede apreciar la opinión del personal docente e investigador que imparte docencia en el grado de Ciencias de la Actividad Física y del Deporte sobre la importancia que les dan a distintos aspectos de las editoriales a la hora de considerarlas editoriales de reconocido prestigio.

Tabla 1: Criterios de calidad de las editoriales (0 Nada importante, 5 Muy importante)

\begin{tabular}{|c|c|c|c|c|}
\hline & $\begin{array}{l}\text { Funcionarios } \\
\qquad(n=67)\end{array}$ & $\begin{array}{c}\text { Interinos/ } \\
\text { Contratados }(n=75)\end{array}$ & $\begin{array}{l}\text { Total PDI } \\
(n=142)\end{array}$ & $\begin{array}{l}P \\
\text { valor }\end{array}$ \\
\hline Que existan comités de lectura especializados & $4.29 \pm 0.89$ & $3.88 \pm 1.31$ & $4.09 \pm 1.13$ & .046 \\
\hline Revisores Externos & $4.53 \pm 0.79$ & $4.40 \pm 0.74$ & $4.47 \pm 0.76$ & .867 \\
\hline Información sobre el proceso de publicación de originales & $3.93 \pm 1.14$ & $3.73 \pm 1.20$ & $3.83 \pm 1.17$ & .414 \\
\hline Que la editorial sea especializada & $3.61 \pm 1.30$ & $3.70 \pm 1.38$ & $3.65 \pm 1.34$ & 659 \\
\hline Que la editorial tenga colecciones especializadas & $3.31 \pm 1.29$ & $3.19 \pm 1.35$ & $3.26 \pm 1.31$ & 686 \\
\hline $\begin{array}{l}\text { Que la editorial tenga un número elevado de } \\
\text { publicaciones en Ciencias del Deporte }\end{array}$ & $3.50 \pm 1.26$ & $3.48 \pm 1.11$ & $3.49 \pm 1.19$ & .403 \\
\hline Trayectoria continuada de la editorial & $3.97 \pm 0.90$ & $3.79 \pm 0.95$ & $3.89 \pm 0.93$ & .815 \\
\hline Reseñas positivas en las mejores revistas del área & $3.77 \pm 1.09$ & $3.88 \pm 0.98$ & $3.82 \pm 1.04$ & .234 \\
\hline Elevado número de títulos & $2.60 \pm 1.20$ & $2.58 \pm 1.10$ & $2.59 \pm 1.15$ & .310 \\
\hline Editor de relevancia en la editorial o colección & $3.38 \pm 1.06$ & $3.15 \pm 1.22$ & $3.28 \pm 1.14$ & 649 \\
\hline $\begin{array}{l}\text { Que las agencias de evaluación valoren positivamente a } \\
\text { esa editorial }\end{array}$ & $3.90 \pm 1.09$ & $3.86 \pm 1.12$ & $3.88 \pm 1.10$ & .974 \\
\hline Presencia en librerías nacionales y extranjeras & $3.64 \pm 1.21$ & $3.48 \pm 1.27$ & $3.56 \pm 1.24$ & .333 \\
\hline Presencia en bibliotecas nacionales y extranjeras & $3.74 \pm 1.21$ & $3.44 \pm 1.33$ & $3.60 \pm 1.27$ & .131 \\
\hline Elevada presencia en bases de datos internacionales & $4.25 \pm 0.95$ & $4.38 \pm 0.78$ & $4.31 \pm 0.88$ & .216 \\
\hline Marketing editorial & $2.99 \pm 1.31$ & $2.76 \pm 1.44$ & $2.88 \pm 1.37$ & .477 \\
\hline Proyección internacional de la editorial & $3.82 \pm 1.07$ & $3.77 \pm 1.00$ & $3.80 \pm 1.03$ & .942 \\
\hline Presentación formal & $3.03 \pm 1.21$ & $2.68 \pm 1.41$ & $2.86 \pm 1.31$ & .086 \\
\hline Que la editorial publique en formatos digitales & $2.96 \pm 1.32$ & $2.76 \pm 1.46$ & $2.86 \pm 1.38$ & .255 \\
\hline
\end{tabular}

Dentro de los funcionarios destaca la importancia que le dan a que las editoriales tengan revisores externos, que tengan comités de lectura especializados y que tengan una elevada presencia en bases de datos internacionales, coincidiendo estos aspectos con los aspectos que eligieron los profesores no funcionarios. Por otro lado, tanto los funcionarios como los interinos/contratados señalan que los aspectos menos importantes a la hora de considerar una editorial de reconocido prestigio son el elevado número de títulos, el marketing editorial, que publiquen en formato digital y la presentación formal. Al preguntarles sobre qué criterio 
es el más importante, el $28.6 \%$ respondieron que tener revisores externos, el $15.2 \%$ que existan comités especializados de lectura y el $12.4 \%$ que tengan una elevada presencia en bases de datos internacionales.

Con respecto a diferencias entre los dos grupos en todos los criterios, los funcionarios presentaron valores superiores a los interinos/contratados, si bien sólo se encuentran diferencias estadísticamente significativas en la existencia de comités de lectura especializados. En la tabla 2 se puede observar el porcentaje de PDI que han considerado a las siguientes editoriales en Ciencias de la Actividad Física y del Deporte, como editoriales de "reconocido prestigio".

Tabla 2: Editoriales españolas en Ciencias de la Actividad Física y del Deporte consideras de "Reconocido Prestigio"

\begin{tabular}{|l|c|c|c|c|}
\hline & $\begin{array}{c}\text { Interinos/ } \\
\text { contratados } \\
(n=67)\end{array}$ & $\begin{array}{c}\text { Funcionarios } \\
(n=75)\end{array}$ & Total PDI $(n=142)$ & $P$ Valor \\
\hline Alianza & $20.9 \%$ & $22.7 \%$ & $21.8 \%$ & .799 \\
\hline Cátedra & $9.0 \%$ & $17.3 \%$ & $13.4 \%$ & .143 \\
\hline EMPanamericana & $52.2 \%$ & $56.0 \%$ & $54.2 \%$ & .653 \\
\hline Graó & $31.3 \%$ & $28.0 \%$ & $29.6 \%$ & .663 \\
\hline Inde & $88.1 \%$ & $69.3 \%$ & $78.2 \%$ & .007 \\
\hline Librería deportiva ESM & $20.9 \%$ & $22.7 \%$ & $21.8 \%$ & .799 \\
\hline Paidotribo & $85.1 \%$ & $69.3 \%$ & $76.8 \%$ & .027 \\
\hline Piramide & $9.0 \%$ & $9.3 \%$ & $9.2 \%$ & .938 \\
\hline Síntesis & $20.9 \%$ & $44.0 \%$ & $33.1 \%$ & .003 \\
\hline Wanceulen & $55.2 \%$ & $38.7 \%$ & $46.5 \%$ & .048 \\
\hline
\end{tabular}

En la tabla 2 se observa que a nivel nacional las editoriales más elegidas por el profesorado interino de Ciencias de la Actividad Física y del Deporte son Inde y Paidotribo destacando bastante sobre la tercera más elegida que es Wanceulen. Por otro lado, para los profesores funcionarios las editoriales más elegidas fueron: Inde, Paidotribo y EMPanamericana. Si se comparan las editoriales elegidas por cada grupo se encontraron diferencias estadísticamente significativas en: Inde, Paidotribo, Síntesis y Wanceulen. Siendo Síntesis más elegida por los funcionarios que por los no funcionarios a diferencia de las otras tres que fueron más elegidas por los no funcionarios. Al analizar la opinión general, únicamente Inde, Paidotribo y EMPanamericaca, son consideradas por más de la mitad de encuestados como editoriales de "Reconocido prestigio". En la tabla 3 se observan las editoriales internacionales que han sido elegidas por los PDI de ciencias del deporte como editoriales de reconocido prestigio.

Tabla 3: Porcentaje de elección de las editoriales internacionales más importantes por parte del PDI en Ciencias de la Actividad Física y el Deporte

\begin{tabular}{|l|c|c|c|c|}
\hline & $\begin{array}{c}\text { Interinos/ } \\
\text { contratados }(n=67)\end{array}$ & Funcionarios $(n=75)$ & Total PDI $(n=142)$ & $P$ Valor \\
\hline Blackwell & $11.9 \%$ & $17.3 \%$ & $14.8 \%$ & .366 \\
\hline Elsevier & $73.1 \%$ & $58.1 \%$ & $65.2 \%$ & .061 \\
\hline Human Kinetics & $77.6 \%$ & $93.3 \%$ & $85.9 \%$ & .007 \\
\hline John Willey and son & $6.0 \%$ & $13.5 \%$ & $9.9 \%$ & .135 \\
\hline McGrawHill & $52.2 \%$ & $60.3 \%$ & $56.4 \%$ & .338 \\
\hline Pearson & $17.9 \%$ & $12.2 \%$ & $14.9 \%$ & .338 \\
\hline Routledge & $39.4 \%$ & $53.3 \%$ & $46.8 \%$ & .098 \\
\hline Sage & $14.9 \%$ & $18.7 \%$ & $16.9 \%$ & .553 \\
\hline Spinger & $34.3 \%$ & $24.0 \%$ & $28.9 \%$ & .175 \\
\hline Taylor and Francis & $68.7 \%$ & $61.3 \%$ & $64.8 \%$ & .362 \\
\hline
\end{tabular}


A nivel internacional las editoriales más valoradas por el profesorado interino de Ciencias de la Actividad Física y del Deporte son Human Kinetics, Elsevier y Taylor and Francis. Los profesores funcionarios valoran más las editoriales Human Kinetics, McGrawHill y Taylor and Francis. Si se compara las editoriales elegidas por cada grupo se encuentran diferencias estadísticamente significativas solo en Human Kinetics, siendo la editorial con un porcentaje más alto de todas con casi la totalidad de los funcionarios eligiendo esta editorial como la editorial más importante del área de Ciencias de la Actividad Física y el Deporte.

\section{DISCUSIÓN}

La publicación de libros es el medio de transmisión de resultados de investigación más importante en el área de Humanidades y Ciencias Sociales (Giménez-Toledo, 2016; Hicks, 2004). Esto se contradice con el valor que habitualmente se le aplica a la hora de realizar las evaluaciones de la actividad científica por parte de las agencias de evaluación del personal docente e investigador universitario. Para Giménez-Toledo (2016) algunas de las causas de esta infravaloración son la imposibilidad de abordar una evaluación cualitativa de los libros, la escasez de indicadores y la ausencia de procedimientos claros para la evaluación, estas causas provocan que la valoración de los libros sea mínima. En este sentido el objetivo del presente estudio es conocer por un lado las características que el profesorado de Ciencias de la Actividad Física y el Deporte considera que son importantes en una editorial para considerarla "editorial prestigiosa" o de reconocido prestigio, y por otro lado conocer cuáles son para ellos las editoriales nacionales e internacionales más importantes.

Con respecto a los indicadores de calidad de las editoriales, los aspectos más importantes obtenidos en este trabajo son: que las editoriales tengan revisores externos, que tengan comités de lectura especializados y que tengan una elevada presencia en bases de datos internacionales. Estos datos coinciden con los obtenidos por Giménez-Toledo y Tejada-Artigas (2015) en su trabajo sobre las editoriales en educación, donde los profesores encuestados escogieron como indicadores más importantes que utilizaran un sistema de evaluación con revisores externos, especialización de la editorial y el prestigio y el sistema de difusión y distribución editorial. Por otro lado, estos mismos autores en otro trabajo sobre editoriales especializadas en comunicación, biblioteconomía y documentación (Giménez-Toledo y Tejada-Artigas, 2012) obtuvieron como resultado de la encuesta a 3045 profesores universitarios los siguientes indicadores de calidad: trayectoria continuada de la editorial, sistema de evaluación mediante revisores externos, presencia en bibliotecas especializadas, presencia en bases de datos internacionales.

Se puede observar la importancia sobre todo de los procesos de evaluación mediante revisores externos y la presencia en bases de datos internacionales como los dos aspectos más importantes a la hora de evaluar la calidad de una editorial. Con respecto a la importancia de los procesos de revisión externa, Mangas-Vega y Gómez-Díaz (2015) en los resultados de su trabajo, sobre los criterios de calidad y la autopublicación, exponen que la editorial tiene una gran carga de responsabilidad en los indicadores de calidad del contenido, lo que hace ver la necesidad de establecer controles de contenido como la revisión por pares. Por otra parte, Quintas-Froufe (2015) realiza una comparación de los criterios de evaluación de las distintas agencias españolas, obteniendo como resultado que los indicadores de calidad usados por las tres agencias analizadas (ANECA, ANEP y CNEAI), en el área de Ciencias Sociales son: el número y calidad de las citas, el prestigio internacional de la editorial y las reseñas en revistas científicas de su área. En esta línea, analizando los resultados de este mismo trabajo con los resultados obtenidos por otros autores en diferentes áreas de las ciencias sociales se puede observar una tendencia en la que los criterios seleccionados para valorar la calidad de las editoriales son los mismos que se usan para las revistas científicas. En este sentido, Giménez-Toledo y Tejada-Artigas, (2015) en las conclusiones de su trabajo ya exponen esta misma idea destacando también la falta de información y transparencia sobre varios de los procesos editoriales como por ejemplo en la selección de manuscritos. La selección de estos criterios puede obedecer a la búsqueda de la objetivación, y cuantificación de la evaluación de la calidad de las editoriales para poder igualarlas en importancia a las evaluaciones de los artículos científicos a la hora de realizar la evaluación para solicitar las distintas acreditaciones.

Otro aspecto para reflexionar, que deriva directamente de la selección de determinados criterios, es el porqué de la selección de éstos. En este sentido, la elección de revisores externos puede responder a la búsqueda de validez de contenido. En esta línea Delgado (2017a), indica que, si las revistas se han convertido en un instrumento de certificación y validación del conocimiento, es imprescindible que este proceso de validez se realice por medio de evaluaciones eficientes, que sólo se obtienen por medio de una evaluación justa e imparcial de la misma comunidad científica. Por este mismo motivo es necesario la aplicación de revisiones por pares en los libros. 
Por otro lado, al comparar las editoriales españolas escogidas en primer lugar por los profesores encuestados en este estudio, y las editoriales tanto del área de Educación Física y Deportiva como del área de Didáctica de la Expresión Corporal recogidas en el Publish Scholar Metrics, se aprecia que en ambos casos la editorial Inde es la más valorada. De igual forma se aprecia, en las primeras posiciones, las editoriales Paidotribo y Wanceulen así como Panamericana. Sin embargo, estas coincidencias no se aprecian al analizar el índice de SPI (Scholarly Publishers Indicators), debido a que en este índice las editoriales de Ciencias de la Actividad Física y del Deporte son incluidas en la sección de Educación, en donde las editoriales como Síntesis, Grao, Catedra, Alianza, y Pirámide, que además de Ciencias de la Actividad Física y el Deporte editan libros de educación, tienen valores más destacados que las editoriales específicas de esta área.

En este mismo sentido al analizar las editoriales internacionales, los PDI de CAFD valoran principalmente las editoriales de Human Kinetics (especifica de Ciencias de la Actividad Física y del Deporte), seguido de Elsevier y Taylor and Francis, mientras que dentro del de SPI (Scholarly Publishers Indicators), son más valoradas Elsevier y Taylor and Francis (editoriales generalistas) que Human Kinetics. Al igual que en las editoriales nacionales, las editoriales específicas de Ciencias de la Actividad Física y el Deporte son menos valoradas que las generalistas al estar incluidas en la sección de Educación del SPI. Por lo que la creación de una sección de esta área sería una gran aportación para facilitar las evaluaciones de la producción científica. Finalmente cabe señalar que la única alusión que las diferentes agencias de valoración (ANECA, CNEAI) hacen en relación al valor de una obra en relación a su editorial, es que: a) sea de una editorial de reconocido prestigio, sin embargo no indican ningún criterio que ayude identificar dicho aspecto; b) que la editorial sea de primer o segundo cuartil de SPI, cuando el producto SPI, dejo de actualizase en el año 2014; y c) o que cuente con el sello CEA (Calidad de Edición Académica), sello promovido por la Unión de Editoriales Universitarias Españolas (UNE) y avalado por la Agencia Nacional de Evaluación de la Calidad y Acreditación (ANECA) y la Fundación Española para la Ciencia y la Tecnología (FECYT), que ha sido recientemente creado, y apenas se ha resuelto la primera convocatoria, por lo que pocas editoriales lo han obtenido.

Dentro del área de las Ciencias de la Actividad Física y del Deporte, los datos del presente estudio pueden ser una ayuda para conocer o para objetivar y clarificar el concepto de "editorial prestigiosa" en esta área y pueda servir de orientación a la hora de evaluar las publicaciones. En cualquier caso, queda bastante por avanzar en la evaluación de editoriales y en la búsqueda de indicadores, que al igual que pasa con la evaluación de los artículos, sea más objetiva y útil a la hora de realizar una evaluación de la actividad investigadora en general, y en las Ciencias de la Actividad Física y el Deporte en particular. En este sentido se aprecian dos nuevas aportaciones: 1) por un lado la necesidad de ampliar los criterios para realizar una adecuada evaluación de la ciencia añadiendo a los habituales otros criterios como la evaluación cualitativa, métricas alternativas, citas abiertas, criterios que permitan valorar el impacto social, etc. (Giménez-Toledo, 2018); 2) por otro lado la necesidad de que las instituciones evaluadoras valoren la utilidad de la evaluación y fomenten que sea una evaluación en busca de mejorar la calidad de la ciencia, y no permita procesos como los que señala Delgado (2017b) "se empieza evaluando lo que se hace, y se termina haciendo lo que se evalúa". Finalmente es necesario matizar que los resultados del presente estudio proceden de la opinión que tienen los profesores universitarios de ciencias del deporte españoles sobre diferentes aspectos de las editoriales. En ningún caso, se ha pretendido valorar la calidad de cada una de las editoriales, sino la opinión que tiene el profesorado universitario tanto de los diferentes criterios de lo que es una adecuada editorial, así como detectar las principales editoriales, con la finalidad de que la opinión de los evaluados, pueda ser un criterio más a la hora de realizar evaluaciones adecuadas y justas.

\section{CONCLUSIONES}

De acuerdo a los resultados de este estudio, y de su discusión y análisis se pueden extraer las siguientes tres conclusiones principales:

1) Las editoriales españolas de reconocido prestigio más valoradas por el pdi de Ciencias de la Actividad Física y el Deporte son: Inde, Paidotribo y Wanceulen; 2) Las editoriales internacionales de reconocido prestigio más valoradas por el pdi de Ciencias de la Actividad Física y el Deporte son: Human Kinetics, Elsevier y Taylor and Francis; y 3) Los aspectos más importantes que tiene que tener una editorial para ser considerada de reconocido prestigio son: tener revisores externos, tener comités de lectura especializados y tener una elevada presencia en bases de datos internacionales.

\section{REFERENCIAS}

Anderson, B. y G. Feist, Transformative Science: a New Index and the Impact of Non-Funding, Private Funding, and Public Funding, Social Epistemology, 31(2), 130-151 (2017)

ANECA, Sello de Calidad en Edición Académica, ANECA (2018) 
Buela-Casal, G. y J.C. Sierra, Criterios, Indicadores y Estándares para la Acreditación de Profesores Titulares y Catedráticos de Universidad, Psicothema, 19(4), 557-591 (2007)

Chacón, S., J.A. Pérez-Gil., F.P. Holgado y L. Ruiz, Evaluación de la Calidad Universitaria: Validez de Contenido, Psicothema, 13(2), 94-301 (2001)

Chang, T. C. y H. Wang, A Multi Criteria Group Decision-making Model for Teacher Evaluation in Higher Education Based on Cloud Model and Decision Tree, Eurasia Journal of Mathematics, Science \& Technology Education, 12(5), 1243-1262 (2016)

Codina, L., Evaluación de la Ciencia: tan Necesaria como Problemática, El profesional de la información, 25(5), 715-719 (2016)

Delgado, E., Evaluar Revistas Científicas: un Afán con mucho Presente y Pasado e Incierto Futuro, En: Revistas científicas: situación actual y retos de futuro, 73-103, Universitat de Barcelona, España (2017a)

Delgado, E., La Edición Académica a Examen: Índices de Impacto, Rankings y Agencias de Evaluación, En VII Jornada Digital, Ecosistema, proyección e impacto del libro académico, Unión de Editoriales Españolas, (2017b)

ESPAÑA, Ley Orgánica 6/2001, de 21 de Diciembre, de Universidades, BOLETIN OFICAL DEL ESTADO, № 307,24 Diciembre (2001)

ESPAÑA, Real Decreto 415/2015, de 29 de Mayo, por el que se modifica el Real Decreto 1312/2077, de 5 de Octubre, por el que se establece la acreditación nacional para el acceso a los cuerpos docentes universitarios. BOLETIN OFICAL DEL ESTADO, 144, 17 de Junio (2015)

Giménez-Toledo, E. y C. Tejada-Artigas, Proceso de Publicación, Calidad y Prestigio de las Editoriales Científicas en Educación, Educación XXI, 18(1), 17-44 (2015)

Giménez-Toledo, E. y C. Tejada-Artigas, Valoración de Editoriales Especializadas en Comunicación, Biblioteconomía y Documentación: Encuesta a Profesores e Investigadores, El Profesional de la Información 21(1), 50-62, (2012)

Giménez-Toledo, E. y J. Mañana-Rodríguez, Is there Agreement on the Prestige of Scholarly Book Publishers in the Humanities? DELPHI over survey results (2017)

Giménez-Toledo, E., C. Tejada-Artigas y J. Mañana-Rodríguez, Evaluation of Scientific Books' Publishers in Social Sciences and Humanities: Results of a Survey, Research Evaluation, 22 (1), 64-77 (2012)

Giménez-Toledo, E., La Evaluación de las Humanidades y de las Ciencias Sociales en Revisión, Revista Española de Documentación Científica, 208, 41 (3) (2018)

Giménez-Toledo, E., Malestar, Los Investigadores ante su Evaluación, Iberoamericana, Madrid (2016)

Grupo de Evaluación de la Ciencia y la Comunicación Científica (EC3), Publisher Scholar Metrics (2017)

Hicks, D., The Four Literatures of Social Science, En: Moed, H.F.W. Glänzel y U. Schmoch, Handbook of quantitative science and technology research, 473-496, Springer, Netherlands (2004)

Jarvis, A., Introducing Thomson Reuters Books Citation Index, Editors' Bulletin, 6(3), 102-103 (2010)

Larrán-Jorge, M., B. Escobar-Pérez y E. García-Meca, El Sistema de Acreditación Nacional: la Opinión de los Profesores Universitarios de Contabilidad, Revista Española de Documentación Científica, 36(3), e015 (2013)

Mangas-Vega, A. y R. Gómez-Díaz, Los Criterios de Calidad y la Autopublicación, Anales de Documentación 18(2), 1-13 (2015)

Quintas-Froufe, N., Indicadores de Calidad de las Publicaciones Científicas en el Área de Ciencias Sociales en España: un Análisis Comparativo entre Agencias Evaluadoras, Revista de Investigación Educativa, 34(1), $259-272$ (2015)

Salado, J., Criterios para la Evaluación de la Calidad del Personal Docente e Investigador Universitario en Ciencias de la Actividad Física y el Deporte, Tesis Doctoral, Murcia, Universidad de Murcia, España (2017)

Sierra, J.C., G. Buela-Casal, M. Bermúdez y P. Santos-Iglesias, Análisis Transaccional del Sistema de Evaluación y Selección del Profesorado Universitário, Interciencia, 33(4), 251-257 (2008)

Tejedor, F.J., Evaluación del Profesorado Universitario: Enfoque Metodológico y Algunas Aportaciones de la Investigación, Estudios sobre Educación, 16, 79-102 (2009)

The Times Higher Education, The World University Ranking (2017)

Torres-Salinas, D. y E. Delgado-López-Cózar, Cobertura de las Editoriales Científicas del Book Citation Index en Ciencias Sociales y Humanidades: ¿La Historia se Repite?, Anuario thinkEPI, 7, 110-113 (2013)

Torres-Salinas, D., N. Robinson-García, E. Jiménez-Contreras y E. Delgado, Towards a Book Publishers Citation Reports. First approach using the Book Citation Index, Revista Española de Documentación Científica, 35(4), 615-624 (2012)

Verleysen, F. y T. Engels, A Label for Peer-reviewed Books, Journal of the Association for Information Science and Technology, 64(2), 428-430 (2013)

Weir, J., Quantifying Test-Retest Reliability Using the Intraclass Correlation Coefficient and the SEM, The Journal of Strength \& Conditioning Research, 19(1), 231-240 (2005)

Zuccala, A., R. Guns, R. Cornacchia, y R. Bod, Can We Rank Scholarly Book Publishers? A Bibliometric Experiment with the Field of History, Journal of the Association for Information Science and Technology, 66(7), 1333-1347 (2015) 